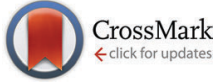

Cite this: Phys. Chem. Chem. Phys., 2016, 18, 31312

Received 26th August 2016, Accepted 31st October 2016 DOI: $10.1039 / \mathrm{c} 6 \mathrm{cp} 05909 \mathrm{k}$

www.rsc.org/pccp

\section{Preferential solvation and ion association properties in aqueous dimethyl sulfoxide solutions $\dagger$}

\author{
Anand Narayanan Krishnamoorthy, Johannes Zeman, Christian Holm and \\ Jens Smiatek*
}

\begin{abstract}
We study the solvation and the association properties of ion pairs in aqueous dimethyl sulfoxide (DMSO) solution by atomistic molecular dynamics (MD) simulations. The ion pair is composed of two lithium and a single sulfonated diphenyl sulfone ion whose properties are studied under the influence of different DMSO concentrations. For increasing mole fractions of DMSO, we observe a non-ideal behavior of the solution as indicated by the derivatives of the chemical activity. Our findings are complemented by dielectric spectra, which also verify a complex DMSO-water mixing behavior. In agreement with these results, further simulation outcomes reveal an aqueous homoselective solvation of the ion species which fosters the occurrence of pronounced ion association constants at higher DMSO mole fractions. The consequences of this finding are demonstrated by lower ionic conductivities for increasing concentrations of DMSO.
\end{abstract}

\section{Introduction}

Ion pairing is an ubiquitous effect in concentrated salt solutions ${ }^{1-3}$ which has some important consequences for complex fluids. ${ }^{4-11}$ The formation of ion aggregates significantly influences dynamic properties like ionic conductivities or diffusivities. Hence, specifically in terms of electrochemical applications, the suppression and modification of ion pair formation is highly desirable. ${ }^{12}$ In general, one can distinguish between contact and solvent-separated ion pairs, ${ }^{1,3,13,14}$ while the mechanisms leading to the formation of these aggregates are still under debate.,14,15 Previous results demonstrated that the solvent itself as well as the solvent chemical activity ${ }^{1,16,17}$ strongly influence many effects, e.g. the formation of ion pairs, ${ }^{1,3}$ the occurrence of specific ion effects ${ }^{18-21}$ and the ion condensation behavior around macromolecules. ${ }^{22-30}$

Based on these findings, one can assume that tunable mixtures provide a controllable influence on the ion pairing mechanism. In this sense, an often discussed concept for binary solutions in order to minimize the number of ion pairs is preferential solvation. ${ }^{31}$ One can distinguish between homoselective solvation, where both ion species are dissolved by the same compound of the solution, and heteroselective solvation where the solvation shells around the ions differ. A typical

Institute for Computational Physics, University of Stuttgart, D-70569 Stuttgart, Germany. E-mail: smiatek@icp.uni-stuttgart.de; Fax: +4971168563658; Tel: +4971163757

$\dagger$ Electronic supplementary information (ESI) available. See DOI: 10.1039/c6cp05909k example for homoselective solvation is the dissolution of $\mathrm{CaCl}_{2}$ in water-methanol mixtures, ${ }^{32}$ where both ion species are preferentially solvated by water molecules. In contrast, silver nitrate in acetonitrile-water mixtures reveals a heteroselective solvation. $^{33}$

A well established empirical framework to understand preferential solvation was proposed by the donor number concept. ${ }^{31,34}$ Herewith, the ability of the solvent to dissolve cations can be quantified. In more detail, solvents with a high donor number usually accumulate around cations, whereas solvents with a high acceptor number prefer the solvation of anions. Notably, the implications of the donor number concept for polyelectrolytes in water, DMSO and chloroform were recently demonstrated by experiments and simulations. ${ }^{35,36}$ The results of these studies indicated that DMSO with a high donor number (DN) fosters the occurrence of free alkali ions, and thus provides a lower association constant compared to water. Therefore, it can be assumed that the usage of binary mixtures for increasing ion dissociation behavior can be regarded as a possible option to improve the ion transport efficiency in electrochemical devices.

In this article, we study the ion pairing behavior between two lithium $\left(\mathrm{Li}^{+}\right)$and a single sulfonated diphenyl sulfone ion $\left(\mathrm{SDS}^{2-}\right)^{30,36}$ in presence of binary DMSO-water mixtures. The corresponding chemical structure of $\mathrm{SDS}^{2-}$ is depicted in Fig. 1. Polymeric forms of $\mathrm{SDS}^{2-}$ and modifications thereof ${ }^{37-40}$ were discussed to be used as building materials for polymer electrolyte membranes in electrochemical applications. ${ }^{35,36}$ The presence of 

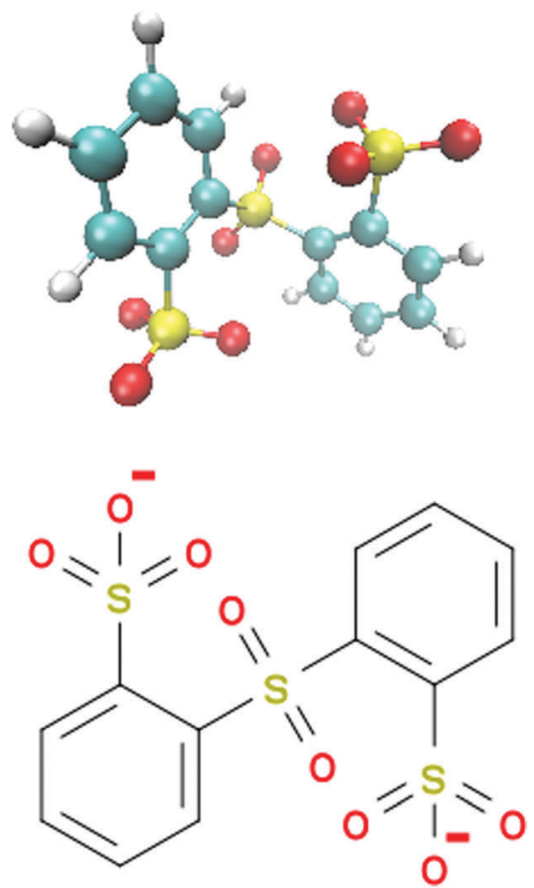

Fig. 1 Molecular snapshot (top) and chemical structure (bottom) of the sulfonated diphenyl sulfone ion $\left(\mathrm{SDS}^{2-}\right)$.

extremely electron-poor poly(phenylene) backbones containing sulfone-units in combination with a large number of sulfonic acid groups allows for high thermal stability and significant ion conductivities. ${ }^{30,35,37,38}$ Another advantage of these polymers is given by their high solubility in water and in a broad range of apolar solvents. ${ }^{36,39}$ In fact, the benefit of non-aqueous solvents rectifies some problems known for ordinary polymer electrolyte membranes in aqueous solution like low working temperatures or low solubility, which can be improved by adding co-solvents such as DMSO. $^{35,41}$

In this article, we exclusively focus on the solvation of a single ion pair in DMSO-water mixtures to avoid the influence of long range electrostatic interactions and possible unwanted correlation effects between like-charged ions. The study of the binary mixture without solutes reveals a significant non-ideal behavior. Moreover, the interpretation of the dielectric spectra indicates a complex cluster formation between DMSO and water molecules in good agreement with experimental results. Further results verify the appearance of a pronounced ion pairing effect with increasing mole fractions of DMSO. Indeed, the simulation outcomes evidence a homoselective solvation of both ion species by water molecules. Our findings imply that homoselective solvation significantly influences the ion association behavior of polyelectrolytes. The presence of solvents with different donor numbers to increase ionic conductivities in terms of electrochemical applications might therefore be questionable and depends on the chosen solvents. Moreover, a previous publication ${ }^{42}$ reported a strong influence of DMSO-water mixtures on the conformational behavior of linear hydrocarbon chains. It can be therefore assumed that the complex interplay between solvents, co-solvents and solutes is more complicated than expected and far from being fully understood. As our study demonstrates, non-ideal solution effects due to interacting solvent molecules and solvent-solute interactions cannot be easily described by simple theoretical concepts. All our numerical results are in good agreement with previous experimental and simulation studies. ${ }^{41,43-46}$

The remainder of this article is organized as follows. In the next two sections we present the theoretical background and the numerical details. The simulation results are presented in the fourth section. We briefly conclude and summarize in the last section.

\section{Theoretical background}

Over the last centuries, several approaches were developed to study non-ideal solution effects. A standard approach to describe non-ideality is given by the chemical activity ${ }^{47,48}$

$$
a_{\alpha}=\exp \left(\mu_{\alpha}-\mu_{\alpha}^{*} / R T\right)
$$

with the chemical potential $\mu_{\alpha}$ of species $\alpha$, the standard chemical potential $\mu_{\alpha}^{*}$, the molar gas constant $R$, and the temperature $T$. Herewith, the deviation from an ideal behavior can be expressed by the activity coefficient ${ }^{47,48}$

$$
\gamma_{\alpha}=\frac{a_{\alpha}}{x_{\alpha}}
$$

with the mole fraction $x_{\alpha}$ of species $\alpha$ in the solution. The presence of an ideal solution is expressed by $\gamma_{\alpha}=1$ whereas non-ideal behavior can be found for $\gamma_{\alpha}<1$. The derivative of the chemical activity is defined as

$$
a_{\alpha \alpha}=\frac{\partial \ln a_{\alpha}}{\partial \ln \rho_{\alpha}},
$$

which can be calculated in terms of the Kirkwood-Buff theory ${ }^{49-52}$ via

$$
a_{\alpha \alpha}=\frac{1}{1+\rho_{\alpha}\left(G_{\alpha \alpha}-G_{\alpha \beta}\right)},
$$

where $G_{\alpha \alpha}$ and $G_{\alpha \beta}$ denote the Kirkwood-Buff integrals

$$
G_{\alpha \beta}=4 \pi \int_{0}^{\infty} r^{2}\left(g_{\alpha \beta}(r)-1\right) \mathrm{d} r
$$

for solvent $\alpha$ and co-solvent $\beta$ molecules in terms of the radial distribution functions $g(r)$ and the number density $\rho_{\alpha}$. With regard to eqn (4), it becomes clear that an ideal mixture is given by $a_{\alpha \alpha}=1$ due to the condition $G_{\alpha \beta}=G_{\alpha \alpha}$ whereas deviations from an ideal behavior are represented by $a_{\alpha \alpha} \neq 1$ and hence $G_{\alpha \beta} \neq G_{\alpha \alpha}$. The values of the Kirkwood-Buff integrals can be interpreted as excess volumes for the individual species compared to an ideal solution as a reference. It has to be noted that the Kirkwood-Buff theory was originally formulated for the grand canonical $\mu V T$ ensemble ${ }^{49-52}$ whereas the applicability for computer simulations was in detail discussed in ref. 53. Hence, approximate Kirkwood-Buff integrals at large but finite distances $r_{\mathrm{c}}$ can be even obtained for $N V T$ (constant temperature) or $N p T$ (constant temperature and constant pressure) simulations. ${ }^{52}$ 
The presence of a preferential solvation mechanism can be detected by the calculation of the local/bulk partition coefficient ${ }^{54-56}$ which reads

$$
K_{\alpha}(r)=\frac{\left(\left\langle n_{\alpha}(r)\right\rangle /\left\langle n_{\beta}(r)\right\rangle\right)}{\left(n_{\alpha}^{\text {tot }} / n_{\beta}^{\text {tot }}\right)},
$$

where $\left\langle n_{x}(r)\right\rangle$ denotes the average number of solvent ( $x=\alpha$, water) or co-solvent molecules ( $x=\beta$, DMSO) within a distance $r$ to the solute and $n_{x}^{\text {tot }}$ the total number of solvent or co-solvent molecules in the simulation box. At short distances of usually $r \leq 0.5 \mathrm{~nm}$, a preferential solvation mechanism by solvent molecules $\alpha$ results in $K_{\alpha}(r)>1$ whereas preferential exclusion can be detected according to $K_{\alpha}(r)<1$.

The occurrence of ion pairs is studied by the normalized cumulative number distribution function or distance-dependent association constant ${ }^{36}$

$$
\theta(R)=\frac{4 \pi}{N_{\mathrm{c}}} \rho_{\mathrm{c}} \int_{0}^{R} r^{2} g_{\text {ic }}(r) \mathrm{d} r
$$

with the number $N_{\mathrm{c}}$ and the number density $\rho_{\mathrm{c}}$ of counterions and the radial distribution functions $g_{\text {ic }}(r)$ between co-ions (i) and counterions (c). A particular interesting value for the association constant in a pure solvent is given for $R=l_{\mathrm{B}}$, with $l_{\mathrm{B}}$ being the Bjerrum length $l_{\mathrm{B}}=e^{2} / 4 \pi \varepsilon_{0} \varepsilon_{\mathrm{r}} k_{\mathrm{B}} T$ with the Boltzmann constant $k_{\mathrm{B}}$, the elementary charge $e$ and the dielectric constant $\varepsilon_{\mathrm{r}}$. It has to be noted that the continuum concept of Bjerrum lengths is only well defined for a solution with a constant global value for $\varepsilon_{\mathrm{r}}{ }^{57,58}$

The dielectric constant of the solution without ions can be calculated by

$$
\varepsilon_{\mathrm{r}}=1+\frac{4 \pi}{3} \frac{\left\langle\vec{M}_{\mathrm{tot}^{2}}{ }^{2}\right\rangle}{V k_{\mathrm{B}} T},
$$

where $\left\langle\vec{M}_{\text {tot }}{ }^{2}\right\rangle$ denotes the average of the squared net total dipole moment of molecules in the simulation box of volume $V .^{59}$ In presence of charged species and periodic boundary conditions, eqn (8) is difficult to apply and one instead has to calculate the autocorrelation function of the total current or the individual currents in the system. ${ }^{60,61}$ Thus, the frequencydependent total conductivity can be written as

$$
\sigma(\omega)=\frac{1}{3 V k_{\mathrm{B}} T}\left\langle\overrightarrow{j_{\mathrm{T}}}(t) \vec{j}_{\mathrm{T}}\left(t_{0}\right)\right\rangle_{\omega}
$$

with the total current $\vec{j}_{\mathrm{T}}$ for angular frequencies $\omega=2 \pi \nu$. The brackets $\langle\cdot\rangle_{\omega}$ denote the Fourier-Laplace transformation of the autocorrelation function reading

$$
\left\langle\overrightarrow{j_{\mathrm{T}}}(t) \overrightarrow{\dot{j}_{\mathrm{T}}}\left(t_{0}\right)\right\rangle_{\omega}=\int_{t_{0}}^{\infty}\left\langle\overrightarrow{j_{\mathrm{T}}}(t) \overrightarrow{j_{\mathrm{T}}}\left(t_{0}\right)\right\rangle e^{\mathrm{i} \omega t} \mathrm{~d} t,
$$

to yield

$$
\varepsilon(\omega)=1+\frac{4 \pi i \sigma(\omega)}{\omega}
$$

as an expression for the static dielectric permittivity after evaluating the real part in the limit $\varepsilon_{\mathrm{r}}=\lim _{\omega \rightarrow 0} \Re(\varepsilon(\omega)) .{ }^{61}$ The real part of the dielectric spectra ("permittivity") is denoted by $\varepsilon^{\prime}(\omega)$ while the negative imaginary part ("loss") is represented by $\varepsilon^{\prime \prime}(\omega)$ such that both terms contribute to the frequencydependent dielectric permittivity $\varepsilon(\omega)=\varepsilon^{\prime}(\omega)-i \varepsilon^{\prime \prime}(\omega)$. Moreover, one can also determine the individual contributions or crosscorrelations for the different constituents of the solution in eqn (9) by taking into account the distinct currents $\vec{j}_{x}$ with $x \in\{\alpha, \beta, i, c\}$. The detailed analysis procedure for the dielectric spectra is discussed in the supplementary material. Finally, the total ionic conductivity $\sigma=\sigma(\omega=0)$ can be calculated by

$$
\lim _{t \rightarrow \infty}\left\langle\Delta \vec{M}_{j}^{2}(t)\right\rangle=2\left\langle\vec{M}_{j}^{2}\left(t_{0}\right)\right\rangle+6 V k_{\mathrm{B}} T \sigma t,
$$

which can be interpreted as the mean squared displacement of the collective translational dipole moment in the simulation box. The so-called Einstein-Helfand conductivity $\sigma$ provides a reliable description of the true conductivity, since it includes ionic correlations that are neglected in the Nernst-Einstein description, which estimates conductivities based on diffusion coefficients. We refer the reader to ref. 60-63 and the supplementary material for more details on the calculation of the ionic conductivity and the dielectric spectra in different systems.

\section{Simulation details}

We studied the properties of two lithium ions $\left(\mathrm{Li}^{+}\right)$and a single molecular sulfonated diphenyl sulfone ion $\left(\mathrm{SDS}^{2-}\right)$ in various compositions of DMSO-water mixtures. A snapshot and the chemical structure of $\mathrm{SDS}^{2-}$ are depicted in Fig. 1. Recent numerical and experimental results revealed interesting ion condensation effects for sulfonated diphenyl sulfones which motivated our choice for the study of this specific ion pair. ${ }^{30,36}$ All-atom molecular dynamics simulations were performed with the GROMACS 4.5.5 software package. ${ }^{64}$ The topologies and force fields for $\mathrm{SDS}^{2-}$, DMSO and $\mathrm{Li}^{+}$were modeled by Generalized Amber Force Fields (GAFF) ${ }^{65,66}$ using ACPYPE ${ }^{67}$ in combination with the TIP3P water model. ${ }^{68}$ It was shown in ref. 69 and 70 that the dynamic and static properties of a broad variety of solvents, including DMSO, are well reproduced by GAFF parameters in agreement with our own consistency checks. A previous publication ${ }^{30}$ with the same force field combination for $\mathrm{SDS}^{2-}$ and sodium ions in presence of TIP3P water reported reasonable results for the ion association behavior in good agreement with experimental findings. In order to check the general validity of our force field combination, we also tested different water models like the TIP4P water ${ }^{68}$ in combination with OPLS/ $\mathrm{AA},{ }^{71}$ which gave comparable results. Two simulation sets of DMSO-water mixtures were performed. One set of simulations in presence of the $\mathrm{SDS}^{2-}$ and two lithium ions and another set for the study of pure DMSO-water mixtures. For pure DMSOwater mixtures, we simulated mole fractions between $x_{\mathrm{DMSO}}=$ 0.1-0.9. In contrast, preferential solvation and association properties of ion pairs were studied for mole fractions between $x_{\text {DMSO }}=0.1-0.5$.

Electrostatic interactions for all systems were calculated by the Particle Mesh Ewald method ${ }^{72}$ with a Verlet cutoff scheme and a short-range radius of $1 \mathrm{~nm}$. The same cutoff scheme was 
also used for the calculation of the Lennard-Jones interactions in combination with dispersion corrections. The Fourier grid spacing was $0.16 \mathrm{~nm}$ and all bonds were constrained by the LINCS algorithm. ${ }^{73}$ The time step in all simulations was 2 fs. All systems were equilibrated for $5 \mathrm{~ns}$ at $300 \mathrm{~K}$ and 1 bar pressure by using the Nose-Hoover thermostat and the Parrinello-Rahman barostat. $^{74,75}$ The initial box size was $(4 \times 4 \times 4) \mathrm{nm}^{3}$. All pure solvent mixtures were simulated for 20 ns whereas the systems with ions were simulated for $50 \mathrm{~ns}$ at $300 \mathrm{~K}$ and 1 bar ( $N p T$ ensemble). In total, we performed more than $10.75 \mu \mathrm{s}$ of simulation time for the calculation of the dielectric spectra, which were compared to the results of our production runs to ensure statistical accuracy (the minimum simulation time for a system was $2.14 \mu \mathrm{s}$ ). More details on the number of solvent molecules and the resulting average box lengths are presented in the supplementary material.

\section{Results}

\subsection{Aqueous DMSO solutions without ions}

We first study the properties of a binary DMSO-water mixture without ions. In fact, the properties of DMSO-water solutions were already discussed in a series of publications. ${ }^{42-46,76-86}$ As a starting point, we analyze the dynamic properties of the solvent molecules. The results for the center-of-mass diffusion coefficients are presented in Fig. 2. For pure water and pure DMSO $\left(x_{\mathrm{DMSO}}=0\right.$ and 1 , respectively), we obtained diffusion coefficients of $D_{\mathrm{cm}}=(0.79 \pm 0.01) \times 10^{-5} \mathrm{~cm}^{2} \mathrm{~s}^{-1}$ (DMSO) and $D_{\mathrm{cm}}=(5.91 \pm 0.01) \times 10^{-5} \mathrm{~cm}^{2} \mathrm{~s}^{-1}$ (water) which are in reasonable agreement with previous experimental findings and simulation results $\left(D_{\mathrm{cm}}=0.73 \times 10^{-5} \mathrm{~cm}^{2} \mathrm{~s}^{-1} \text { (DMSO, exp. }\right)^{87}$ and $D_{\mathrm{cm}}=5.88 \times 10^{-5} \mathrm{~cm}^{2} \mathrm{~s}^{-1}$ (TIP3P, sim. $)^{88}$ ). Minimum diffusion coefficients for both components can be found for DMSO mole fractions between $x_{\text {DMSO }}=0.4-0.6$. Thus, the diffusivity of water molecules dramatically decreases about one order whereas the effects on DMSO are less pronounced. It was already discussed

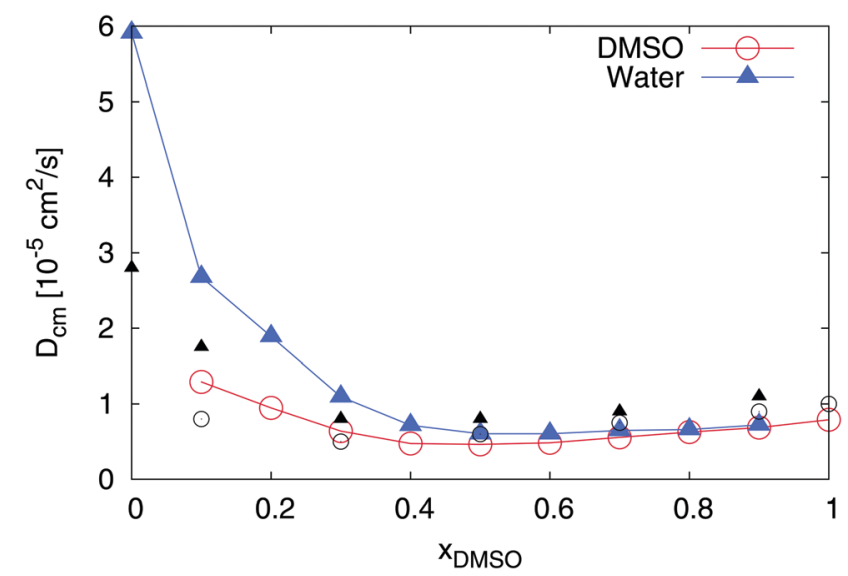

Fig. 2 Diffusion coefficient for DMSO (blue triangles) and water molecules (red circles) in a DMSO-water mixture for different mole fractions $x_{\text {DMSO. All }}$ error bars are within symbol size and black symbols denote experimental results $^{41}$ for DMSO (black circles) and water (black triangles) at $305 \mathrm{~K}$. in previous publications that the observed minimum diffusion coefficients indicate a non-ideal behavior of the mixture, which can be mainly attributed to the formation of pronounced DMSO-water pairs. ${ }^{4,46}$ Furthermore, the observed diffusion coefficients for all molar fractions of DMSO roughly coincide with experimental findings ${ }^{41}$ as the data in Fig. 2 suggests. Hence, also in experiments, a minimum diffusivity between $x_{\text {DMSO }}=0.4-0.6$ becomes visible.

However, the most important indicator for a non-ideal solution behavior is demonstrated by the value of the chemical activity. Therefore, we calculated the derivative of the chemical activity $a_{\alpha \alpha}$ in accordance to eqn (4) for both individual components. As depicted in Fig. 3, water reveals a nearly ideal behavior for all mole fractions with values $a_{\alpha \alpha} \approx 1$. In fact, a value of $a_{\alpha \alpha}=1$ can be directly attributed to the equivalence of the water-water and water-DMSO Kirkwood-Buff integrals (eqn (4)). In contrast, DMSO shows a highly non-ideal behavior with the largest value $a_{\beta \beta} \approx 13.5$ at $x_{\text {DMso }}=0.5$. The large value of $a_{\beta \beta}$ indicates that the value of the Kirkwood-Buff integral for DMSO-DMSO pairs is smaller than the value for the DMSOwater integral according to $G_{\beta \alpha}>G_{\beta \beta}$. Hence, a larger excess molecule number around DMSO as defined by ${ }^{52}$

$$
N_{\alpha}^{\mathrm{xs}}=\rho_{\alpha} G_{\beta \alpha}
$$

can be observed for water when compared to DMSO molecules. Therefore, it can be concluded that DMSO preferentially interacts with water molecules in agreement with previous findings. ${ }^{44-46}$ The corresponding implications for the dynamic properties in terms of cross-correlations between DMSO and water will be discussed in the next sections.

In addition to the thermodynamic properties, we also calculated the dielectric constant $\varepsilon_{\mathrm{r}}$ for different mole fractions $x_{\text {DMSo }}$ by evaluating eqn (11) in the limit of $\omega \rightarrow 0$. The corresponding results are depicted in Fig. 4. The black line corresponds to an ideal solution with $\varepsilon_{\mathrm{r}}^{\text {id }}=\varepsilon_{\mathrm{r}}^{\mathrm{H}_{2} \mathrm{O}}\left(1-x_{\text {DMSO }}\right)+$ $\varepsilon_{\mathrm{r}}^{\mathrm{DMSO}} x_{\text {DMSO }}$ with the dielectric constants $\varepsilon_{\mathrm{r}}^{\mathrm{H}_{2} \mathrm{O}}=95.32$ and $\varepsilon_{\mathrm{r}}^{\mathrm{DMSO}}=55.54$ as found in our simulations for the pure solvents.

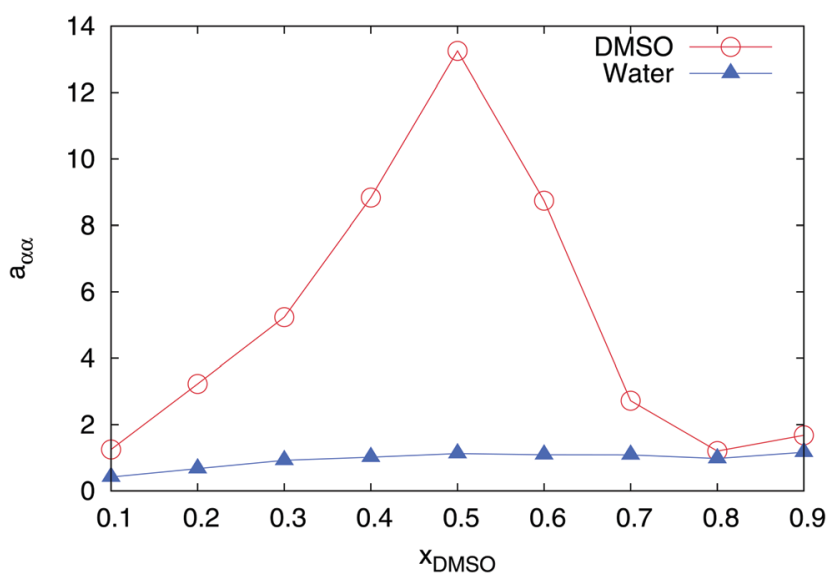

Fig. 3 Derivative of the chemical activity $a_{\alpha \alpha}$ for DMSO (red circles) and water (blue triangles) in a DMSO-water mixture for different mole fractions $x_{\text {DMSO }}$. All error bars are within symbol size. 


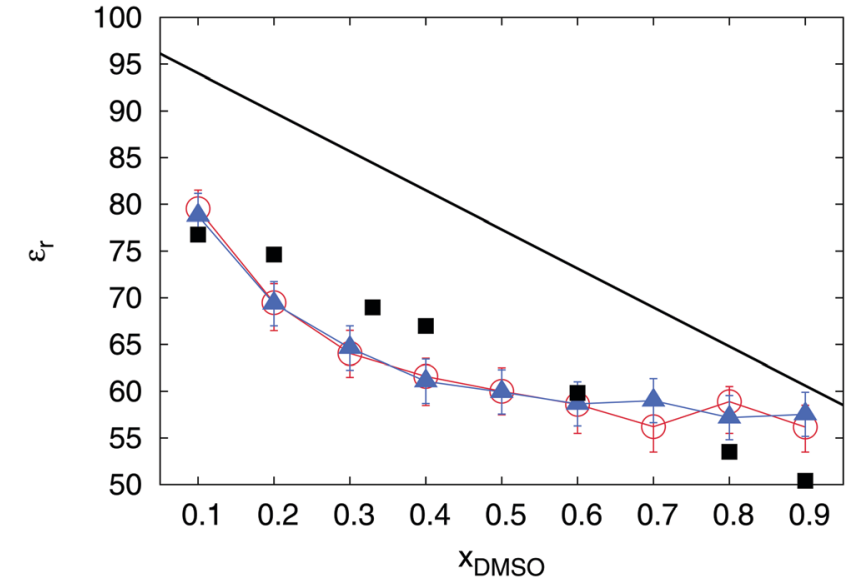

Fig. 4 Dielectric constant $\varepsilon_{\mathrm{r}}$ of the solution with standard deviations for different mole fractions $x_{\mathrm{DMSO}}$ as calculated by eqn (11). The black line is the theoretical result for an ideal solution. The red circles denote the results for the pure DMSO-water mixture in contrast to the blue triangles which represent the outcomes for the DMSO-water solution in presence of $\mathrm{Li}^{+}$and $\mathrm{SDS}^{2-}$ ions. Black squares denote experimental results ${ }^{44}$ at $298 \mathrm{~K}$.

The results for pure TIP3P water agree with previous simulations $\left(\varepsilon_{\mathrm{r}}=96.9\right)^{89}$ whereas the dielectric constant for DMSO slightly deviates from experimental results $\left(\varepsilon_{\mathrm{r}}=47.24\right) .{ }^{90}$ Based on our simulation outcomes, it can be concluded that a direct interaction between water and DMSO molecules due to $\varepsilon_{\mathrm{r}}<\varepsilon_{\mathrm{r}}^{\text {id }}$ is evident such that the resulting total dipole moment is significantly decreased compared with an ideal solution. Thus, all previous findings indicate a complicated interaction between DMSO and water molecules leading to highly non-ideal solution effects for intermediate DMSO mole fractions. Moreover, also experimental results ${ }^{44}$ reveal a decrease of the dielectric constant as the data in Fig. 4 indicate. Although the exact functional form differs from our findings, the range of values for the dielectric constant is roughly comparable.

\subsection{Solvation properties of ion species}

In this subsection, we focus on the solvation properties of the ions. In order to test the validity of our chosen force fields, we calculated the radial distribution function between water molecules and lithium ions in presence of the $\mathrm{SDS}^{2-}$ ion and in pure water. It was discussed that specifically the hydration properties of lithium ions are of significant importance to characterize specific ion binding effects. ${ }^{91}$ The results can be found in the supplementary material. We observed a pronounced first hydration shell around $\mathrm{Li}^{+}$within distances of $0.3 \mathrm{~nm}$. The corresponding coordination number of water molecules by the integration of the radial distribution function to this distance $e^{92}$ yields $N_{\mathrm{H}_{2} \mathrm{O}}=4.72$. The position of the first hydration shell as well as the resulting water coordination number are in good agreement with $a b$ initio MD simulation results ${ }^{93}$ where a coordination number $N_{\mathrm{H}_{2} \mathrm{O}} \approx 5$ was reported. Therefore, we can assume that the influence of lithium ions on its local hydration shell is correctly reproduced by our chosen force field combination.

In order to proceed with the solvation properties of the ions in the DMSO-water mixture, we first study the radial
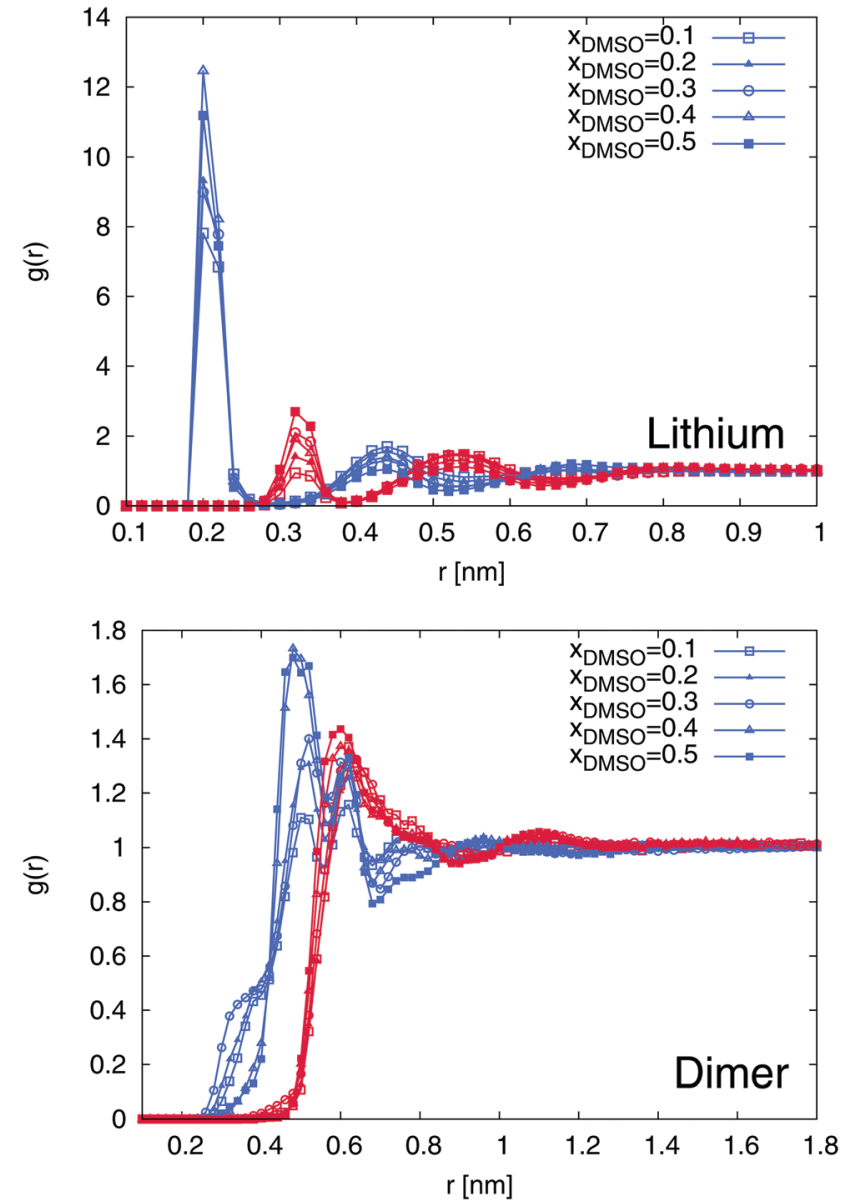

Fig. 5 Radial distribution functions at different mole fractions $x_{\mathrm{DMSO}}$ for the center-of-masses of DMSO and water molecules around lithium ions (top) and $\mathrm{SDS}^{2-}$ (bottom). The results for the different mole fractions are denoted by the symbols described in the legend. Blue lines correspond to water RDFs whereas all red lines represent the radial distribution of DMSO molecules around the individual ions.

distribution functions (RDFs) for DMSO and water molecules around lithium ions and $\mathrm{SDS}^{2-}$. The results are shown in Fig. 5. It becomes evident that the first solvent shell around lithium ions with a maximum value at $r \approx 0.2 \mathrm{~nm}$ is purely composed of water molecules. With higher mole fractions of DMSO, this finding is even more pronounced with regard to slightly increasing values for the water peak. In contrast, the second solvent shell with a peak position at $r \approx 0.35 \mathrm{~nm}$ is formed by DMSO molecules. In total, one can observe five distinct water and DMSO solvent shells within distances $r \leq 0.9 \mathrm{~nm}$. This finding can be mostly rationalized in terms of entropic effects due to the smaller size of the water molecules compared to DMSO in accordance with properties discussed for sizeasymmetric ionic liquids. ${ }^{94,95}$ Noteworthy, the first solvent shell around lithium ions is always occupied by water molecules which contradicts the assumption in terms of the higher donor number for DMSO compared to water $\left(\mathrm{DN}_{\mathrm{DMSO}}=29.8 \mathrm{vs}\right.$. $\left.\mathrm{DN}_{\mathrm{H}_{2} \mathrm{O}}=18.0\right) \cdot{ }^{34}$ Based on our findings, it can be concluded that molecular size effects dominate the preferential solvation 
of lithium ions in contrast to specific chemical details as it was discussed in a previous publication for pure solvents. ${ }^{36}$

A comparable behavior can be found for $\mathrm{SDS}^{2-}$. The first solvent shell is formed by water molecules whereas a mixed second solvent shell composed of DMSO and water molecules can be found at $r \approx 0.65 \mathrm{~nm}$. Interestingly, for larger values of $x_{\mathrm{DMSO}}$, one can observe a significant increase of the first solvent shell peak value for $x_{\mathrm{DMSO}} \geq 0.3$.

The results for the local/bulk partition coefficient in accordance to eqn (6) are shown in Fig. 6 and 7. In order to obtain reliable estimates, we only calculated the values at distances where both solvent species have a minimum coordination number of at least $N_{\alpha} \geq 0.2$. It can be clearly seen that the local/bulk partition DMSO coefficient around SDS ${ }^{2-}$ (bottom of Fig. 6) reveals $K_{\mathrm{DMSO}}(r)<1$ for all distances. Thus, it becomes evident that DMSO is preferentially excluded from $\mathrm{SDS}^{2-}$. This finding is also validated with regard to the strong preferential solvation of $\mathrm{SDS}^{2-}$ by water molecules (bottom of Fig. 7). All values reveal $K_{\mathrm{H}_{2} \mathrm{O}}(r)>1$ at short distances which even increases for higher mole fractions of DMSO. Thus, a preferential solvation of $\mathrm{SDS}^{2-}$ by water molecules is indeed validated.

In addition to $\mathrm{SDS}^{2-}$, lithium ions reveal a comparable solvation behavior (top of Fig. 6 and 7). In accordance to the
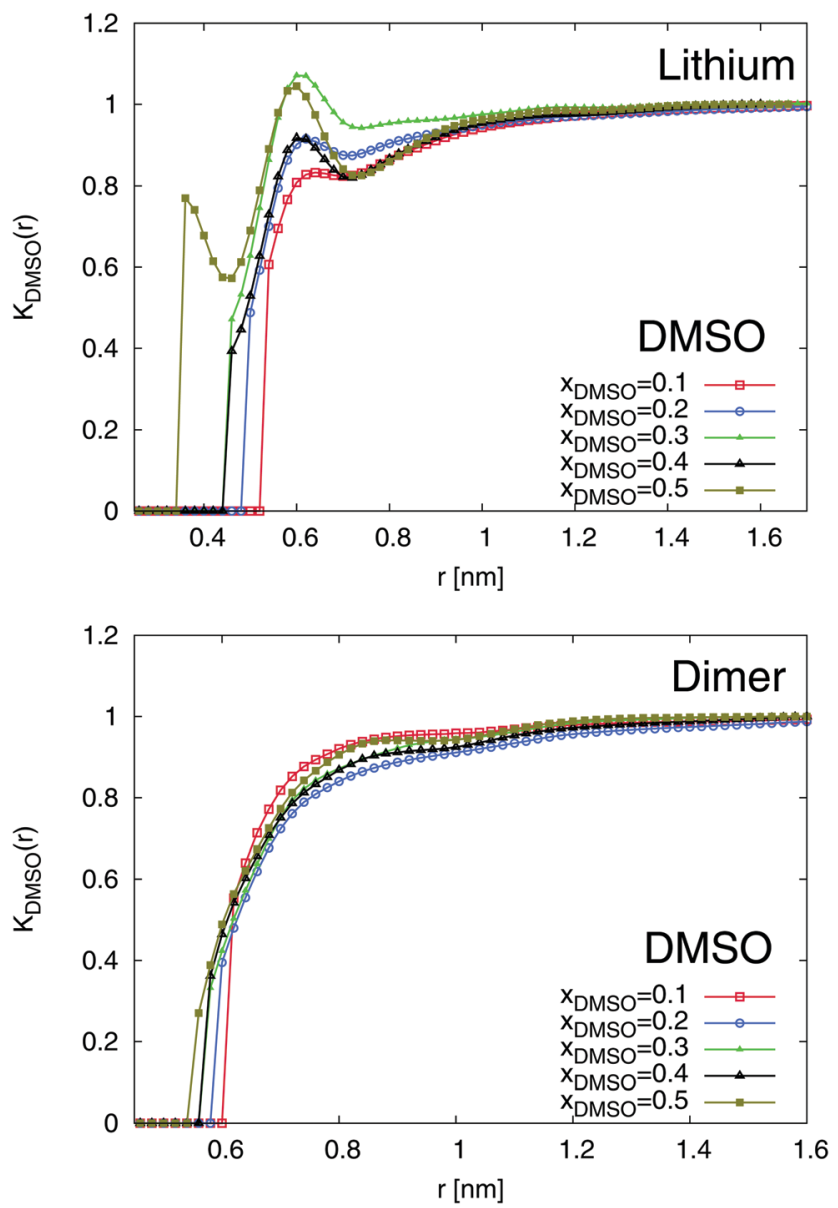

Fig. 6 Local/bulk partition coefficient for DMSO molecules around lithium (top) and the $\mathrm{SDS}^{2-}$ ion (bottom) for different DMSO mole fractions $x_{\mathrm{DMSO}}$.
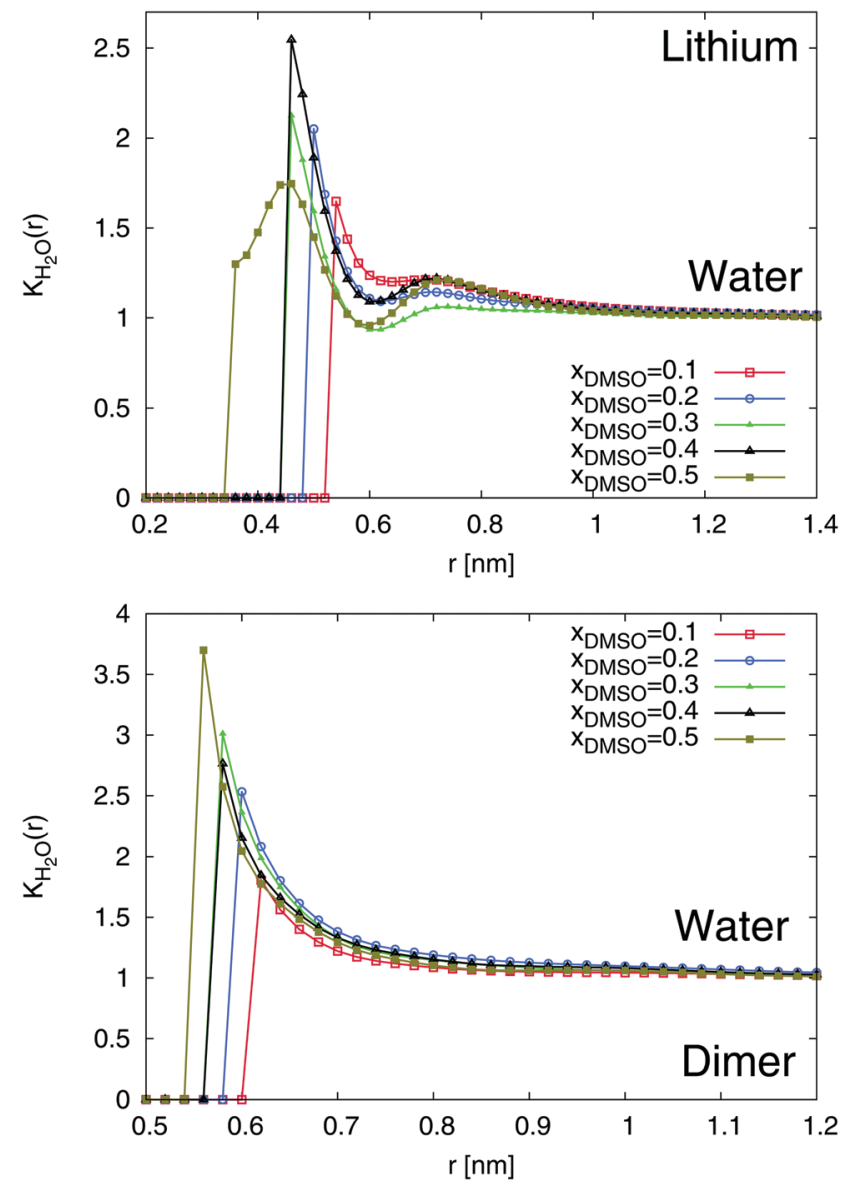

Fig. 7 Local/bulk partition coefficient for water molecules around lithium (top) and the $\mathrm{SDS}^{2-}$ ion (bottom) for different DMSO mole fractions $x_{\text {DMSO. }}$

radial distribution functions shown in Fig. 5, it can be clearly seen that the first solvent shell at short distances is fully occupied by water molecules due to negligible values of $K_{\mathrm{DMSO}}(r) \approx 0$ at $r \leq 0.3 \mathrm{~nm}$. The presence of distinct solvent shells remains also valid for the second pure DMSO shell at $r \approx 0.35 \mathrm{~nm}$. At larger distances, a slightly preferable accumulation of DMSO molecules around $\mathrm{Li}^{+}$in the mixed third solvent shell is indicated by $K_{\mathrm{DMsO}}(r) \geq 1$ at distances $r \geq 0.6$ and DMSO mole fractions $x_{\text {DMSO }} \geq 0.4$ (top of Fig. 6). In conjunction with this finding, large values of $K_{\mathrm{H}_{2} \mathrm{O}}$ around lithium ions can be observed at the same distance indicating that even in the mixed third solvent shell an accumulation of water molecules is preferred (top of Fig. 7). Thus, both ion species are therefore more likely solvated by water molecules which verifies a homoselective solvation mechanism. Indeed, these results reveal the non-applicability of the donor and acceptor number concept for DMSO-water mixtures.

\subsection{Dielectric spectra of aqueous DMSO solutions in presence of ions}

For the study of the dynamic properties of the solution, we calculated the real $\varepsilon^{\prime}(\omega)$ and the negative imaginary part $\varepsilon^{\prime \prime}(\omega)$ of the frequency-dependent dielectric permittivity according to eqn (11). The corresponding procedure for the data analysis 

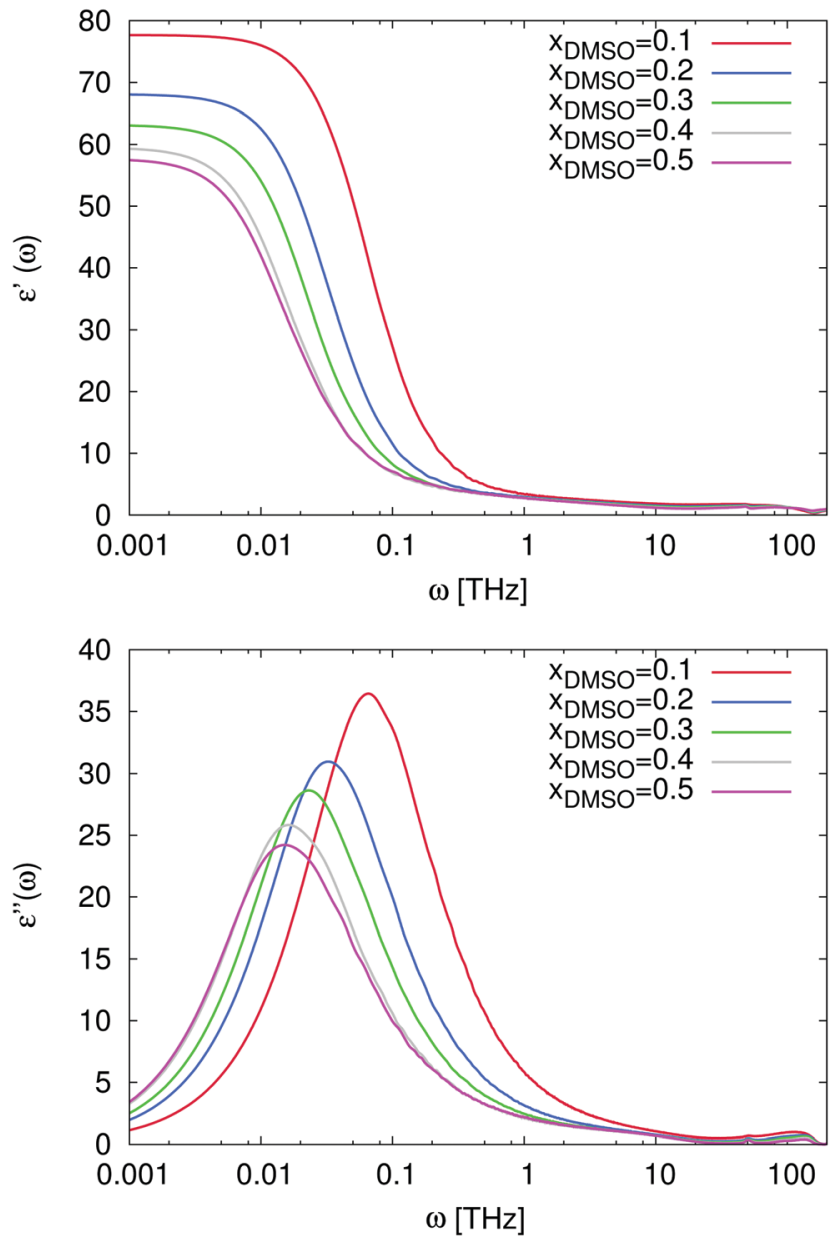

Fig. 8 Top: Frequency-dependent dielectric permittivity $\varepsilon^{\prime}(\omega)$ for mole fractions $x_{\mathrm{DMSO}}=0.1-0.5$ in presence of the ion pair. Bottom: Frequencydependent dielectric loss $\varepsilon^{\prime \prime}(\omega)$ for mole fractions $x_{\mathrm{DMSO}}=0.1-0.5$ in presence of the ions.

is discussed in detail in the supplementary material whereas the results are presented in Fig. 8. The noisy data were slightly smoothed to point out the main properties and the raw data can be found in the supplementary material. As we have already mentioned before, the static dielectric permittivity $\varepsilon^{\prime}(\omega)$ can be estimated for $\omega \rightarrow 0$. One can clearly observe in Fig. 8 , that the resulting values decrease for increasing mole fractions of DMSO in agreement with Fig. 4.

In more detail, the imaginary part of the dielectric spectrum which is shown in the bottom of Fig. 8 reveals some interesting properties as one would expect for non-ideal solutions. Main absorption peaks can be observed between $\omega=0.1 \mathrm{THz}$ and $\omega=0.01 \mathrm{THz}$ for DMSO mole fractions ranging from $x_{\mathrm{DMSO}}=$ 0.1 to $x_{\mathrm{DMSO}}=0.5$. A similar trend for increasing mole fractions with a shift of the absorption peak was also experimentally observed. ${ }^{44}$ The corresponding relaxation times are $\tau \approx 63 \mathrm{ps}$ $\left(\omega=0.1 \mathrm{THz}\right.$ for $\left.x_{\mathrm{DMSO}}=0.1\right)$ and $\tau \approx 630 \mathrm{ps}(\omega=0.01 \mathrm{THz}$ for $\left.x_{\text {DMSO }}=0.5\right)$ in comparison to experimental data ${ }^{44}$ at $298 \mathrm{~K}$ between 22.54 ps $\left(x_{\text {DMSO }}=0.1\right)$ and 53.94 ps $\left(x_{\text {DMSO }}=0.4\right)$. Moreover, one can also observe a small peak at $\omega \approx 100 \mathrm{THz}$ which can be related to pure water modes as discussed in ref. 62 and 96. The absence of this peak for higher DMSO mole fractions complements this assumption and can be explained by librational modes of water which are suppressed with increasing DMSO fractions. In addition, also the far-infrared peak at $\omega=0.1 \mathrm{THz}$ belongs to the hydrogen bond network of water molecules due to the presence of a varying number of hydrogen-bonded neighbors which individually contribute to the low frequency spectrum. ${ }^{96}$ Thus it follows, that for DMSO concentrations between $x_{\mathrm{DMSO}}=0.1-0.5$, the number of hydrogenbonded water neighbors decreases with increasing DMSO concentration and the relaxation time increases in agreement with the observed shift to lower frequencies.

Also the non-ideal behavior of the solution is reflected by the data. In fact, the frequency of the absorption peak at $\omega \approx 0.01 \mathrm{THz}$ remains constant for DMSO mole fractions $x_{\mathrm{DMSO}}=0.4$ and $x_{\text {DMSO }}=0.5$ as it was also experimentally found ${ }^{44}$ while for lower DMSO mole fractions a shift of the main peak to higher frequencies can be observed. As it was discussed in the previous subsections, it can be therefore concluded that the minimum frequency associated with the corresponding peak position can be brought into agreement with the non-ideal behavior of the solution. These results are also in reasonable agreement with our findings for the diffusion coefficient in Fig. 2 and with previous experimental results discussing the occurrence of pronounced DMSO-water interactions. ${ }^{44,45}$ It can be expected that cross-correlation contributions between water and DMSO molecules might significantly contribute to this observation.

In order to study these effects in more detail, we also calculated the individual as well as the cross-correlation contributions of the species in the solution. The corresponding results are presented in Fig. 9. As a first remark, it has to be noted that ionic contributions to the dielectric spectra and their cross-correlations with other compounds are negligible and therefore not shown. For the individual solvent contributions, one can clearly see that the static dielectric permittivity $\varepsilon_{\mathrm{r}}=\varepsilon^{\prime}(\omega=0)$ for mole fractions $x_{\text {DMSO }} \leq 0.3$ is mostly dominated by water molecules. Thus, the corresponding values for water are located in the range between $\varepsilon_{\mathrm{r}}=11.7\left(x_{\mathrm{DMSO}}=0.5\right)$ and $\varepsilon_{\mathrm{r}}=59.5\left(x_{\text {DMSO }}=0.1\right)$ as shown in Table 1. In addition, the individual contributions to the dielectric constant are presented in the bottom of Fig. 9. It can be clearly seen that the contributions of water and DMSO to the dielectric constant decrease or increase, respectively nearly linearly with the DMSO mole fraction. Hence, the contributions of pure water and DMSO to the dielectric constant behave in good agreement with an ideal solution. The non-linear behavior mainly arises from cross-correlations between DMSO and water molecules. The main peak value is located at $x_{\mathrm{DMSO}}=0.4$ indicating the highest amount of DMSO-water correlation effects. In addition, one can recognize that for a DMSO mole fraction between $x_{\text {DMSO }}=0.3-0.4$ all contributions are nearly comparable. Indeed, the crucial interplay between the molecules can be related to the high non-ideality of the solution at these DMSO mole fractions.

The non-ideality is also reflected by the absorption behavior of the solution in terms of the dielectric loss shown in Fig. 10. In comparison to the spectra shown in Fig. 9, also the absorption peaks of $\varepsilon^{\prime \prime}(\omega)$ are strongly dominated by water contributions for 

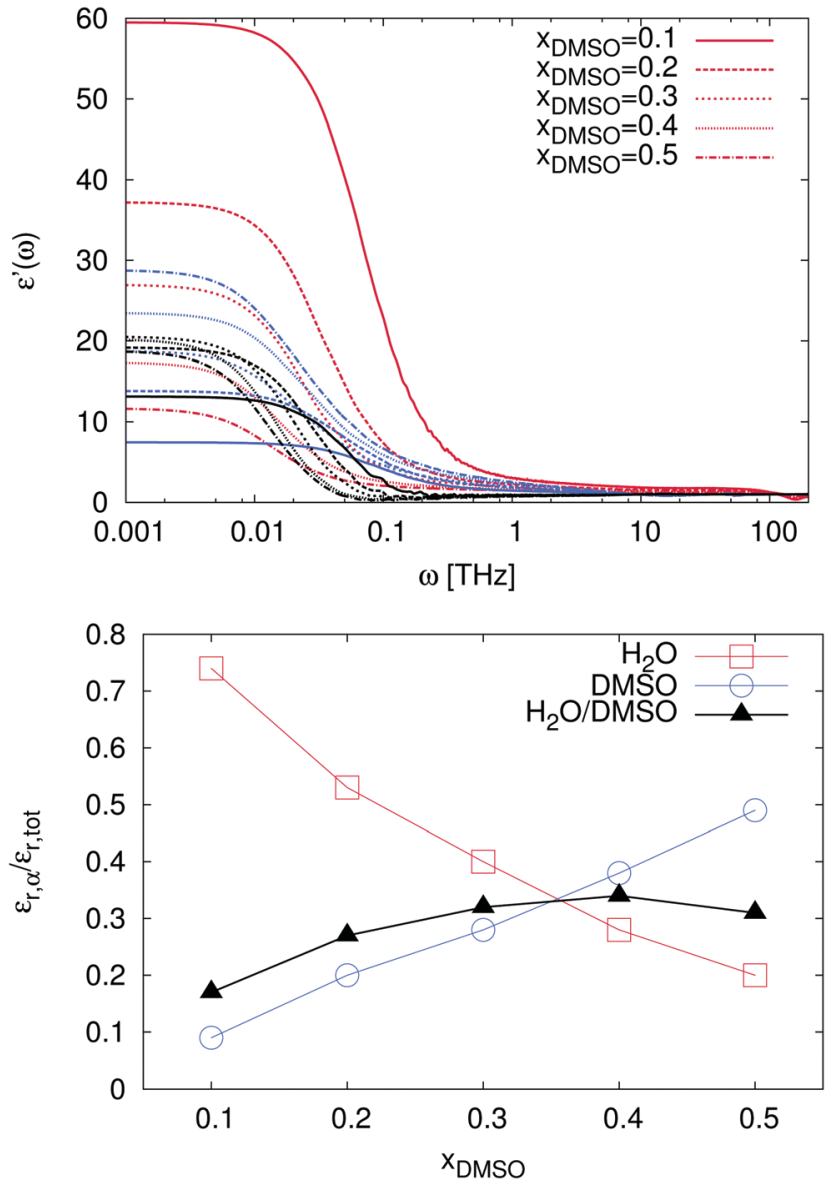

Fig. 9 Top: Contributions to the frequency-dependent dielectric permittivity $\varepsilon^{\prime}(\omega)$ for mole fractions $x_{\mathrm{DMSO}}=0.1-0.5$ in presence of the ions. The contributions of the ions can be neglected due to their low intensity. Red lines denote the results for water contributions, blue lines for DMSO and black lines represent the cross correlation contributions for water and DMSO. Bottom: Relative contributions of the individual species and cross correlation contributions $\varepsilon_{r, \alpha}$ to the resulting total dielectric constant $\varepsilon_{r, t o t}$. Red squares denote the results for water contributions whereas blue circles and black triangles represent the results for pure DMSO and correlated water-DMSO contributions, respectively.

Table 1 Individual and correlated dielectric constants of the solvent compounds for different mole fractions $x_{\mathrm{DMSO}}$. The results for water contributions are denoted by $\varepsilon_{\mathrm{H}_{2}} \mathrm{O}$ whereas $\varepsilon_{\mathrm{DMSO}}$ and $\varepsilon_{\mathrm{H}_{2} \mathrm{O} / \mathrm{DMSO}}$ represent the results for pure DMSO and correlated water-DMSO contributions, respectively

\begin{tabular}{lrrrrr}
\hline$x_{\text {DMSO }}$ & 0.1 & 0.2 & 0.3 & 0.4 & 0.5 \\
\hline$\varepsilon_{\mathrm{H}_{2} \mathrm{O}}$ & 59.5 & 37.2 & 26.7 & 17.4 & 11.7 \\
$\varepsilon_{\text {DMSO }}$ & 7.5 & 13.8 & 18.7 & 23.5 & 28.8 \\
$\varepsilon_{\mathrm{H}_{2} \mathrm{O} / \text { DMSO }}$ & 13.2 & 19.2 & 20.6 & 20.2 & 18.8
\end{tabular}

low DMSO fractions. Interestingly, we observe a shift of the main peak to lower frequencies for both solvents with increasing DMSO fraction. Therefore, it can be concluded that the interaction between DMSO and water molecules directly affect the resulting dynamic properties of water molecules. Moreover, pronounced correlation effects between DMSO and water molecules due to peaks at frequencies between $\omega=0.05-0.01 \mathrm{THz}$ corresponding to

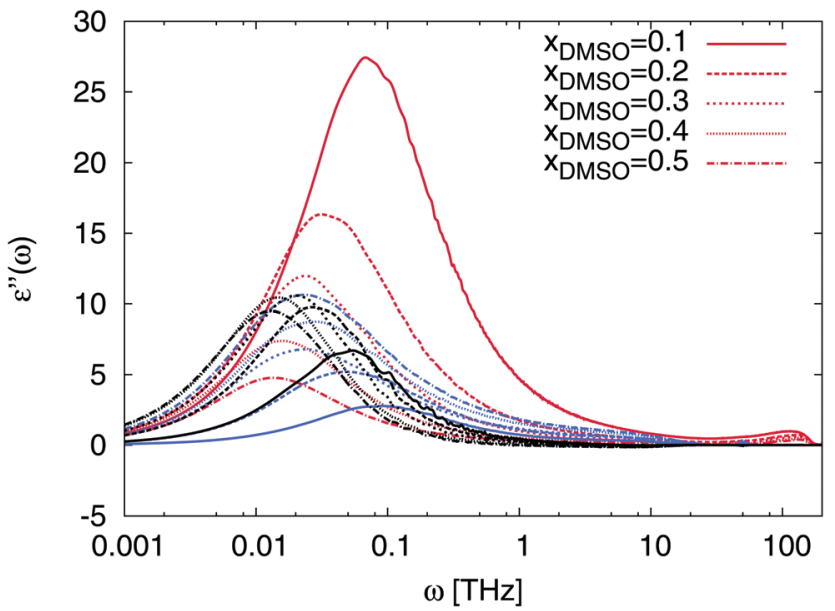

Fig. 10 Contributions to the frequency-dependent dielectric loss $\varepsilon^{\prime \prime}(\omega)$ for mole fractions $x_{\mathrm{DMSO}}=0.1-0.5$ in presence of the ion pair. Red lines denote the results for water contributions, blue lines for DMSO and black lines represent the cross correlation contributions for water and DMSO. The contributions of the ions can be neglected due to their low intensity.

molecular relaxation times between $\tau=125 \mathrm{ps}$ and $\tau=630 \mathrm{ps}$ become visible. For mole fractions between $x_{\text {DMso }}=0.3-0.5$ representing a non-ideal solution, main absorption peaks between $\omega=0.01-0.03 \mathrm{THz}$ can be found which indicates that correlation effects between DMSO and water occur on characteristic time scales between $\tau=200-600 \mathrm{ps}$. Therefore, it can be concluded that DMSO-water interactions result in stable complexes which reorganize at the sub-nanosecond time scale. Compared to pure water, where characteristic time scales occur on picoseconds, ${ }^{97}$ it can be stated that DMSO-water complexes show roughly two orders longer reorganization times. These findings verify the complicated interaction behavior between DMSO and water molecules which directly affect the non-ideality of the solution at specific DMSO mole fractions. A molecular picture of this effect can be established by the analysis of the intermolecular hydrogen bonds. The corresponding hydrogen bond analysis shown in the supplementary material reveals an increasing fraction of water-DMSO hydrogen bonds for higher DMSO mole fractions. A previous publication ${ }^{43}$ discussed this finding as the main reason for the non-ideality of the solution.

\subsection{Ion pairing and ion conductivity}

Finally, we study the formation of ions pairs in aqueous DMSO solutions. Several theories were proposed to describe the amount of ion pairing in aqueous systems (for an overview we refer the reader to ref. 1 and 3). We distinguished between bound pairs and free ions by the introduction of a cutoff radius $R=1.4 \mathrm{~nm}$. Hence, if lithium ions are located within this distance around the charged $-\mathrm{SO}_{3}{ }^{-}$group, we considered them as condensed. Although this distance is quite large, it allows us to point out qualitative trends in the ion pairing process. The value for the cutoff distance can be rationalized with regard to Fig. 6 and 7, where bulk solution behavior for distances $r \geq 1.4 \mathrm{~nm}$ around both ion species can be found. A systematic estimate of the association constant at the Bjerrum length is 


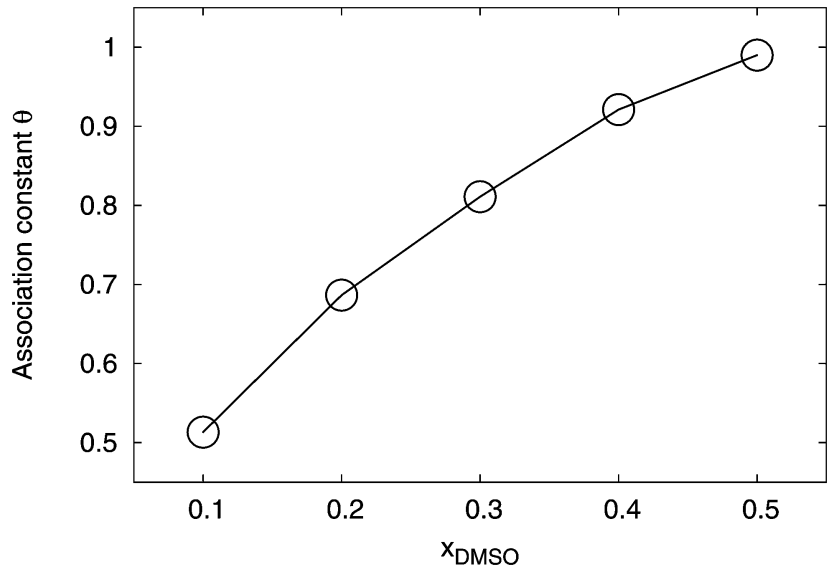

Fig. 11 Association constant of the ion pair as represented by the fraction of condensed counterions $\theta_{c}$ for different mole fractions $x_{\mathrm{D} M S O}$ at $R=1.4 \mathrm{~nm}$. All error bars are within symbol size and the solid line is a guide for the eye.

inapplicable due inhomogeneous solvation shells around the ions which induce a locally varying dielectric constant. The corresponding results for $R=1.4 \mathrm{~nm}$ are presented in Fig. 11 .

Indeed, one can see that the association constant $\theta$, according to eqn (7) which represents the fraction of bound lithium ions, grows non-linearly with the mole fraction of DMSO. This finding remains also valid for variations of the cutoff distances $R$. In more detail, the results for the radial distribution function between the $\mathrm{SDS}^{2-}$ and the lithium ions (data not shown) indicated that the ion association behavior is mostly represented by direct contact pairs ${ }^{1}$ at distances $r \approx 0.2 \mathrm{~nm}$. We compared the results for the association constant to standard theoretical predictions. ${ }^{1,98-100}$ A satisfying agreement with these theories could not be achieved. This might be either due to our limited set of data, inaccuracies of the chosen force fields or the influence of specific solution properties. In more detail, it was discussed in ref. 28 and 58 that specific ion effects as well as locally varying dielectric constants prohibit the usage of standard theories like the Eigen-Fuoss equation ${ }^{1,98}$ or modifications of the Manning-Oosawa counterion condensation theory by a Poisson-Boltzmann cell model approach. ${ }^{99-101}$ All these theories rely on the absence of finite-size effects and the presence of homogeneous continuum solution properties for the dielectric constant. With regard to Fig. 5 , it becomes obvious that our system significantly deviates from these assumptions and reveals pronounced non-idealities, which results in an inapplicability of standard theoretical approaches. ${ }^{28}$ Hence, novel theories have to be developed, e.g. as they were discussed in ref. 19 and 28 in order to establish a reliable prediction of the counterion condensation behavior. More detailed studies in the future will help to shed light at this point. Nevertheless, our results indicate that a significant ion pair formation occurs for higher mole fractions of DMSO which complements the findings for the homoselective solvation behavior.

Furthermore, the ionic conductivity $\sigma$ according to eqn (12) reveals a non-linear decrease for increasing DMSO fractions. The results are presented in Fig. 12. We obtained values between

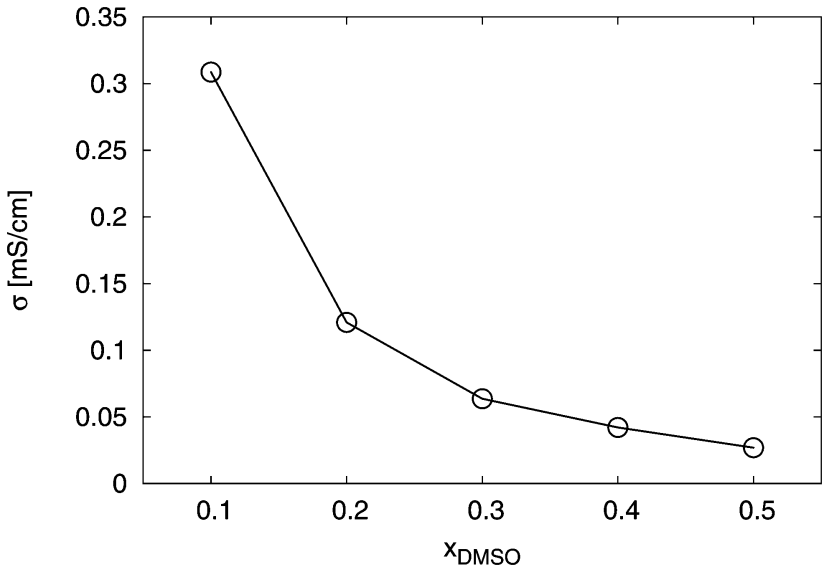

Fig. 12 Total conductivity $\sigma$ for the solution in presence of the ions for

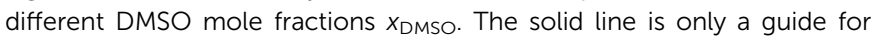
the eye.

$\sigma=0.31 \mathrm{mS} \mathrm{cm}^{-1}$ for $x_{\text {DMSO }}=0.1$ and $\sigma=0.03 \mathrm{mS} \mathrm{cm}^{-1}$ for $x_{\text {DMSO }}=0.5$ with a steep decrease for mole fractions between $x_{\text {DMSO }}=0.1-0.3$. Noteworthy, a comparable decrease of $\sigma$ for a polymeric form of $\mathrm{SDS}^{2-}$ was also recently observed. ${ }^{41}$ Thus, the ionic conductivity decreases by roughly one order for increasing DMSO fractions. Therefore, it can be concluded that large association constants are accompanied by low ionic conductivities. Based on our findings, it becomes evident that a significant fraction of ion pairs decrease the ionic conductivity which is disadvantageous for optimizing ion conductivities in electrochemical applications.

\section{Summary and conclusion}

We studied the properties of ion pairs in binary solvent mixtures of DMSO and water by molecular dynamics simulations. Our results for the derivative of the chemical activity indicate a strong non-ideal behavior of the pure solution without ions for mole fractions between $x_{\text {DMSO }}=0.3-0.5$. These findings are complemented and supported by the analysis of the dielectric spectra. All our results verify a strong water-DMSO interaction for moderate DMSO mole fractions resulting in stable complexes. The presence of these complexes as well as their binding properties significantly influence the dynamic and the static properties of the solution resulting in the strong non-ideal behavior. Moreover, the absence of a preferential heteroselective solvation behavior for the ions is evident. In fact, the first solvation shell around both constituents is purely formed by water molecules. Vice versa, we observed a preferential exclusion of DMSO around both ion species. Hence, with regard to the functionality of DMSO molecules in the mixture and on their action on the solutes, it can be speculated that DMSO reveals similar properties like osmolytes known as protein protectants, e.g. trimethylamine- $\mathrm{N}$-oxide (TMAO), ectoine or hydroxyectoine. ${ }^{97}$ Specifically for these molecules, a preferential exclusion around differently charged solutes was observed. Thus, the strong interaction with the local solvent shell favors their occurrence in bulk solution 
which agrees with the observed behavior for DMSO. In summary, one can assume that heteroselective solvation is mostly influenced by the solvent composition, the specific molecular details of both solvent molecules and their preference to interact with the solute.

In summary, our study indicates that preferential heteroselective solvation of different solutes with regard to varying values for the donor and acceptor numbers fails for aqueous DMSO solutions. Competing effects like preferential exclusion in aqueous solution might dominate, which verifies the complicated interplay between the components of the mixture. A strong indicator is given by the non-ideality of the solution which demonstrates that both solvent molecules strongly interact. Our study presents a first step towards a deeper understanding of the underlying effects. More effort has to be spent for a full understanding of these interesting solution properties.

\section{Acknowledgements}

We thank Andreas Wohlfarth, Klaus-Dieter Kreuer and Frank Uhlig for valuable discussions. Financial funding is gratefully acknowledged from the Deutsche Forschungsgemeinschaft through the SFB 716 and the cluster of excellence "Simulation Technology" (EXC 310).

\section{References}

1 Y. Marcus and G. Hefter, Chem. Rev., 2006, 106, 4585-4621.

2 A. Ciferri and A. Perico, Ionic interactions in natural and synthetic macromolecules, Wiley Online Library, 2012.

3 N. F. A. van der Vegt, K. Haldrup, S. Roke, J. Zheng, M. Lund and H. J. Bakker, Chem. Rev., 2016, 116, 7626-7641.

4 T. Köddermann, C. Wertz, A. Heintz and R. Ludwig, ChemPhysChem, 2006, 7, 1944-1949.

5 H. Weingärtner, Angew. Chem., Int. Ed., 2008, 47, 654-670.

6 R. G. Winkler, M. Gold and P. Reineker, Phys. Rev. Lett., 1998, 80, 3731.

7 A. Naji, S. Jungblut, A. G. Moreira and R. R. Netz, Physica A, 2005, 352, 131-170.

8 H. Boroudjerdi, Y.-W. Kim, A. Naji, R. R. Netz, X. Schlagberger and A. Serr, Phys. Rep., 2005, 416, 129-199.

9 T. Farhat, G. Yassin, S. T. Dubas and J. B. Schlenoff, Langmuir, 1999, 15, 6621-6623.

10 B. Qiao, J. J. Cerdà and C. Holm, Macromolecules, 2010, 43, 7828-7838.

11 M. Vögele, C. Holm and J. Smiatek, J. Chem. Phys., 2015, 143, 243151.

12 K.-D. Kreuer, Chem. Mater., 2013, 26, 361-380.

13 G. Ciccotti, M. Ferrario, J. T. Hynes and R. Kapral, Chem. Phys., 1989, 129, 241-251.

14 P. Ganguly, P. Schravendijk, B. Hess and N. F. van der Vegt, J. Phys. Chem. B, 2011, 115, 3734-3739.

15 T. Hajari, P. Ganguly and N. F. van der Vegt, J. Chem. Theory Comput., 2012, 8, 3804-3809.
16 J. Rösgen, B. M. Pettitt, J. Perkyns and D. W. Bolen, J. Phys. Chem. B, 2004, 108, 2048-2055.

17 J. Rösgen, B. M. Pettitt and D. W. Bolen, Biochemistry, 2004, 43, 14472-14484.

18 Y. Marcus, Chem. Rev., 2009, 109, 1346-1370.

19 W. Kunz, Specific ion effects, World Scientific, 2010.

20 P. Lo Nostro and B. W. Ninham, Chem. Rev., 2012, 112, 2286-2322.

21 P. Jungwirth and P. S. Cremer, Nat. Chem., 2014, 6, 261-263.

22 Y. Zhang and P. S. Cremer, Curr. Opin. Chem. Biol., 2006, 10, 658-663.

23 B. Jagoda-Cwiklik, R. Vácha, M. Lund, M. Srebro and P. Jungwirth, J. Phys. Chem. B, 2007, 111, 14077-14079.

24 M. Lund, R. Vácha and P. Jungwirth, Langmuir, 2008, 24, 3387-3391.

25 B. Hess and N. F. van der Vegt, Proc. Natl. Acad. Sci. U. S. A., 2009, 106, 13296-13300.

26 N. Vlachy, B. Jagoda-Cwiklik, R. Vácha, D. Touraud, P. Jungwirth and W. Kunz, Adv. Colloid Interface Sci., 2009, 146, 42-47.

27 E. A. Algaer and N. F. van der Vegt, J. Phys. Chem. B, 2011, 115, 13781-13787.

28 J. Heyda and J. Dzubiella, Soft Matter, 2012, 8, 9338-9344.

29 J. Paterová, K. B. Rembert, J. Heyda, Y. A. Kurra, H. I. Okur, W. R. Liu, C. Hilty, P. S. Cremer and P. Jungwirth, J. Phys. Chem. B, 2013, 117, 8150-8158.

30 A. Wohlfarth, J. Smiatek, K.-D. Kreuer, S. Takamuku, P. Jannasch and J. Meier, Macromolecules, 2015, 48, 1134-1143.

31 C. Reichardt and T. Welton, Solvents and solvent effects in organic chemistry, John Wiley \& Sons, 2011.

32 H. Schneider and H. Strehlow, Z. Elektrochem., 1962, 66, 309-312.

33 Y. Marcus, J. Chem. Soc., Dalton Trans., 1991, 2265-2268.

34 V. Gutmann, Electrochim. Acta, 1976, 21, 661-670.

35 K.-D. Kreuer, A. Wohlfarth, C. C. de Araujo, A. Fuchs and J. Maier, ChemPhysChem, 2011, 12, 2558-2560.

36 J. Smiatek, A. Wohlfarth and C. Holm, New J. Phys., 2014, 16, 025001.

37 M. Schuster, K.-D. Kreuer, H. T. Andersen and J. Maier, Macromolecules, 2007, 40, 598-607.

38 M. Schuster, C. C. de Araujo, V. Atanasov, H. T. Andersen, K.-D. Kreuer and J. Maier, Macromolecules, 2009, 42, 3129-3137.

39 G. Titvinidze, K.-D. Kreuer, M. Schuster, C. C. de Araujo, J. P. Melchior and W. H. Meyer, Adv. Funct. Mater., 2012, 22, 4456-4470.

40 C. Wang and S. J. Paddison, J. Phys. Chem. A, 2013, 117, 650-660.

41 A. Wohlfarth, Charge carrier formation, mobility and microstructure of sulfonated polyelectrolytes for electrochemical applications, PhD thesis, University of Stuttgart, Germany, 2015, http://dx.doi.org/10.18419/opus-6877.

42 R. Ghosh, S. Banerjee, S. Chakrabarty and B. Bagchi, J. Phys. Chem. B, 2011, 115, 7612-7620.

43 A. Luzar and D. Chandler, J. Chem. Phys., 1993, 98, 8160-8173. 
44 Z. Lu, E. Manias, D. D. Macdonald and M. Lanagan, J. Phys. Chem. A, 2009, 113, 12207-12214.

45 Z. Lu, E. Manias, M. Lanagan and D. Macdonald, ECS Trans., 2010, 28, 11-21.

46 N. Zhang, W. Li, C. Chen and J. Zuo, Comput. Theor. Chem., 2013, 1017, 126-135.

47 P. W. Atkins and J. de Paula, Physical Chemistry, OUP, Oxford, 2010.

48 M. J. Blandamer, J. B. Engberts, P. T. Gleeson and J. C. R. Reis, Chem. Soc. Rev., 2005, 34, 440-458.

49 J. G. Kirkwood and F. P. Buff, J. Chem. Phys., 1951, 19, 774-777.

50 A. Ben-Naim, Statistical thermodynamics for chemists and biochemists, Springer Science \& Business Media, 2013.

51 P. E. Smith, Biophys. J., 2006, 91, 849-856.

52 V. Pierce, M. Kang, M. Aburi, S. Weerasinghe and P. E. Smith, Cell Biochem. Biophys., 2008, 50, 1-22.

53 P. E. Smith, J. Phys. Chem. B, 2004, 108, 18716-18724.

54 E. Courtenay, M. Capp, C. Anderson and M. Record, Biochemistry, 2000, 39, 4455-4471.

55 F. Rodríguez-Ropero and N. F. van der Vegt, J. Phys. Chem. B, 2014, 118, 7327-7334.

56 S. Micciulla, J. Michalowsky, M. A. Schroer, C. Holm, R. von Klitzing and J. Smiatek, Phys. Chem. Chem. Phys., 2016, 18, 5324-5335.

57 W. B. Russel, D. A. Saville and W. R. Schowalter, Colloidal dispersions, Cambridge University Press, 1989.

58 F. Fahrenberger, O. A. Hickey, J. Smiatek and C. Holm, Phys. Rev. Lett., 2015, 115, 118301.

59 M. Neumann, Mol. Phys., 1983, 50, 841-858.

60 J. Caillol, D. Levesque and J. Weis, J. Chem. Phys., 1986, 85, 6645-6657.

61 C. Schröder and O. Steinhauser, J. Chem. Phys., 2009, 131, 114504.

62 M. Sega, S. Kantorovich, C. Holm and A. Arnold, J. Chem. Phys., 2014, 140, 211101.

63 F. Dommert, J. Schmidt, B. Qiao, Y. Zhao, C. Krekeler, L. Delle Site, R. Berger and C. Holm, J. Chem. Phys., 2008, 129, 224501.

64 S. Pronk, S. Páll, R. Schulz, P. Larsson, P. Bjelkmar, R. Apostolov, M. R. Shirts, J. C. Smith, P. M. Kasson, D. van der Spoel, B. Hess and E. Lindahl, Bioinformatics, 2013, 29, 845-854.

65 J. Wang, R. M. Wolf, J. W. Caldwell, P. A. Kollman and D. A. Case, J. Comput. Chem., 2004, 25, 1157-1174.

66 J. Wang, W. Wang, P. A. Kollman and D. A. Case, J. Mol. Graphics Modell., 2006, 25, 247-260.

67 A. W. S. da Silva and W. F. Vranken, BMC Res. Notes, 2012, $5,367$.

68 W. L. Jorgensen, J. Chandrasekhar, J. D. Madura, R. W. Impey and M. L. Klein, J. Chem. Phys., 1983, 79, 926-935.

69 J. Wang and T. Hou, J. Comput. Chem., 2011, 32, 3505-3519.

70 C. Caleman, P. J. van Maaren, M. Hong, J. S. Hub, L. T. Costa and D. van der Spoel, J. Chem. Theory Comput., 2011, 8, 61-74.
71 W. L. Jorgensen, D. S. Maxwell and J. Tirado-Rives, J. Am. Chem. Soc., 1996, 118, 11225-11236.

72 T. Darden, D. York and L. Pedersen, J. Chem. Phys., 1993, 98, 10089-10092.

73 B. Hess, H. Bekker, H. J. C. Berendsen and J. G. E. M. Fraaije, J. Comput. Chem., 1997, 18, 1463-1472.

74 D. Frenkel and B. Smit, Understanding molecular simulation: from algorithms to applications, Academic press, 2001.

75 M. Parrinello and A. Rahman, J. Appl. Phys., 1981, 52, 7182-7190.

76 H. L. Clever and S. P. Pigott, J. Chem. Thermodyn., 1971, 3, 221-225.

77 S. Lam and R. Benoit, Can. J. Chem., 1974, 52, 718-722.

78 P. Fiordiponti, F. Rallo and F. Rodante, Z. Phys. Chem., 1974, 88, 149-159.

79 T. Chan and W. A. van Hook, J. Solution Chem., 1976, 5, 107-123.

80 T. J. Day and G. Patey, J. Chem. Phys., 1999, 110, 10937-10944.

81 I. I. Vaisman and M. L. Berkowitz, J. Am. Chem. Soc., 1992, 114, 7889-7896.

82 I. A. Borin and M. S. Skaf, J. Chem. Phys., 1999, 110, 6412-6420.

83 A. Vishnyakov, A. P. Lyubartsev and A. Laaksonen, J. Phys. Chem. A, 2001, 105, 1702-1710.

84 M. L. Strader and S. E. Feller, J. Phys. Chem. A, 2002, 106, 1074-1080.

85 S. J. Bachmann and W. F. van Gunsteren, J. Phys. Chem. B, 2014, 118, 10175-10186.

86 A. Perera and R. Mazighi, J. Chem. Phys., 2015, 143, 154502.

87 M. Holz, S. R. Heil and A. Sacco, Phys. Chem. Chem. Phys., 2000, 2, 4740-4742.

88 P. Mark and L. Nilsson, J. Phys. Chem. A, 2001, 105, 9954-9960.

89 P. Hochtl, S. Boresch, W. Bitomsky and O. Steinhauser, et al., J. Chem. Phys., 1998, 109, 4927-4937.

90 W. M. Haynes, CRC Handbook of Chemistry and Physics, CRC press, 2014.

91 K. D. Collins, Methods, 2004, 34, 300-311.

92 V. Lesch, A. Heuer, B. R. Rad, M. Winter and J. Smiatek, Phys. Chem. Chem. Phys., 2016, 18, 28403-28408.

93 A. Tongraar, K. R. Liedl and B. M. Rode, Chem. Phys. Lett., 1998, 286, 56-64.

94 V. Lesch, A. Heuer, C. Holm and J. Smiatek, ChemPhysChem, 2016, 17, 387-394.

95 V. Lesch, A. Heuer, C. Holm and J. Smiatek, Phys. Chem. Chem. Phys., 2015, 17, 8480-8490.

96 M. Sega and C. Schröder, J. Phys. Chem. A, 2014, 119, 1539-1547.

97 J. Smiatek, J. Phys. Chem. B, 2014, 118, 771-782.

98 R. M. Fuoss, J. Am. Chem. Soc., 1958, 80, 5059-5061.

99 G. Manning, J. Chem. Phys., 1969, 51, 924-933.

100 F. Oosawa, Polyelectrolytes, Marcel Dekker, New York, 1971.

101 M. Deserno, C. Holm and S. May, Macromolecules, 2000, 33, 199-206. 